\title{
Aplicabilidade de coagulantes a base de tanino em estações de tratamento de água
}

O estabelecimento de práticas que priorizam os aspectos relacionados ao meio ambiente e a saúde pública tem questionado a aplicação de sais de alumínio e ferro como coagulantes em estações de tratamento de água, uma vez que tais produtos possuem em sua composição elementos que podem comprometer o equilíbrio de ecossistemas, além de serem responsáveis por efeitos deletérios a saúde. Esse estudo avaliou a remoção de sólidos suspensos e coloidais da água, em termos de turbidez e cor verdadeira, de um manancial localizado no município de Campina Grande - Paraíba. Foram realizados ensaios de coagulação/decantação/floculação com aparelho Jar test, sendo utilizados coagulantes orgânicos, a base de tanino (Tanfloc SG e Tanfloc SL), e inorgânicos, Cloreto Férrico e Sulfato de Alumínio. Diferentes dosagens e velocidades de sedimentação foram empregadas, além disso, foi verificada a influência desses fatores nos parâmetros obtidos para a água decantada. Os resultados dos ensaios apontaram um melhor desempenho do coagulante orgânico Tanfloc SL, já os métodos estatísticos indicaram independência da velocidade de sedimentação por parte dos coagulantes orgânicos e dependência para os de natureza inorgânica. Desta forma, a adoção do coagulante orgânico tipo Tanfloc SL é uma alternativa factível e sustentável para as estações de tratamento de água.

Palavras-chave: Coagulantes Orgânicos; Coagulantes inorgânicos; Tratamento Sustentável.

\section{Applicability of tannin-based coagulants in water treatment plants}

\begin{abstract}
The establishment of practices that prioritize aspects related to the environment and public health has questioned the application of aluminum and iron salts as coagulants in water treatment plants, since such products have in their composition elements that can compromise the equilibrium of the ecosystems, and they are responsible for deleterious health effects. This study evaluated the removal of suspended and colloidal solids from the water, in terms of turbidity and true color, of a fountainhead located in the city of Campina Grande - Paraiba. Coagulation / settling / flocculation assays were performed with Jar test, using organic coagulants, based on tannin (Tanfloc SG and Tanfloc SL), and inorganic, Ferric Chloride and Aluminum Sulphate. Were used different dosages and sedimentation velocities, in addition, was verified the influence of these factors on the parameters obtained for the decanted water. The results of the tests indicated a better performance of the organic coagulant Tanfloc SL, and the statistical methods indicated independence of the sedimentation rate by the organic coagulants and dependence for the ones of inorganic nature. Therefore, the use of organic coagulant Tanfloc SL is a feasible and sustainable alternative for water treatment plants.
\end{abstract}

Keywords: Organic coagulants; Inorganic coagulants; Sustainable treatment.

Topic: Engenharia Sanitária

Reviewed anonymously in the process of blind peer.
Received: 10/08/2018

Approved: 24/09/2018
Thiago Cabral Nepomuceno (iD)

Universidade Federal de Capina Grande, Brasil

http://lattes.cnpq.br/6513462502498555

http://orcid.org/0000-0002-3452-6647

thiago.nepomucenoesa@gmail.com

Weruska Brasileiro Ferreira (iD

Universidade Estadual da Paraíba, Brasil

http://lattes.cnpq.br/5630172788119332

http://orcid.org/0000-0003-2381-3883

weruska brasileiro@yahoo.com.br

Wiliam de Paiva (D)

Universidade Estadual da Paraíba, Brasil http://lattes.cnpq.br/2612977983185686 http://orcid.org/0000-0003-0220-1247 wpaiva461@gmail.com

\author{
Tarciana Ramos Diniz (iD \\ Universidade Estadual da Paraíba, Brasil \\ http://lattes.cnpq.br/0893437494746183 \\ http://orcid.org/0000-0002-7552-1148 \\ tarciannaramos@gmail.com \\ Whelton Brito dos Santos (iD \\ Universidade Federal de Capina Grande, Brasil \\ http://lattes.cnpq.br/6407528093074339 \\ http://orcid.org/0000-0002-2956-8260 \\ wheltonbrt@gmail.com
}

Referencing this:

NEPOMUCENO, T. C.; FERREIRA, W. B.; PAIVA, W.; DINIZ, T. R.; SANTOS, W. B.. Aplicabilidade de coagulantes a base de tanino em estações de tratamento de água. Revista Ibero Americana de Ciências Ambientais, v.9, n.7, p.110-123, 2018. DOI: http://doi.org/10.6008/CBPC2179-6858.2018.007.0011

DOI: 10.6008/CBPC2179-6858.2018.007.0011 


\section{INTRODUÇÃO}

Dentre os processos e operações pertencentes ao tratamento de água para abastecimento público, a prática da adição de produtos químicos que possam atuar na desestabilização das partículas impuras, facilitando sua posterior remoção, é amplamente difundida e utilizada em boa parte das estações de tratamento de água. A esses produtos químicos, dá-se o nome de coagulantes.

O processo de desestabilização dos coloides e de partículas em suspensão presentes na água é realizado por vários agentes químicos, como sais de alumínio e ferro (SÁNCHEZ-MARTíN et al., 2012), sendo estes os mais aplicados nas estações de tratamento de água (FRANCO et al., 2017). A eficiência desses coagulantes na atenuação dos níveis de materiais particulados, coloidais e substâncias orgânicas presentes na água, aliado aos seus baixos custos de aquisição, são os principais fatores que justificam o predomínio em sua escolha (CORAL, 2009).

A maior conservação e proteção dos recursos naturais, juntamente com resultados de pesquisas científicas, tem questionado a utilização de coagulantes a base de sais metálicos. A principal justificativa para a assertiva anterior está intrinsicamente dependente do fato que coagulantes dessa natureza apresentam em sua constituição compostos que, mesmo em reduzidas concentrações, podem comprometer ecossistemas, promoverem riscos severos a saúde humana, além de interferir negativamente na eficiência das etapas do tratamento convencional de água (OLIVEIRA et al., 2016). Uma alternativa real para substituição dos sais de ferro e alumínio é a utilização de coagulantes de natureza orgânica, como os a base de tanino.

Coagulantes orgânicos a base de tanino, estes extraídos, por exemplo, da árvore Acácia-negra, tem sido objeto de diversas aplicações no âmbito do tratamento de água para abastecimento público. Um exemplo desses coagulantes orgânicos é o Tanfloc, um polímero catiônico que atua em sistemas coloidais, neutralizando cargas e proporcionando a formação de pontes entre essas partículas. Trevisan (2014) realizou ensaios de tratabilidade com a água bruta da estação de tratamento de água (ETA) de Cafezal, localizada no município brasileiro de Londrina, onde comparou o desempenho do Tanfloc SG com o apresentado pelo Sulfato de Alumínio e Cloreto Férrico. A partir dos resultados, o autor pôde definir o Tanfloc SG como forte substituto dos sais de ferro e alumínio, já que o coagulante natural apresentou significativa remoção de parâmetros como cor e turbidez, com valores que respeitam os limites estabelecidos pela legislação vigente (Portaria de consolidação no 5 de 28 de setembro de 2017 do Ministério da Saúde).

Além de mostrar desempenho similar e até mesmo superior aos sais de ferro e alumínio na atenuação de parâmetros relevantes para a potabilização da água, o Tanfloc também pode proporcionar a produção de lodo com elevada biodegrabilidade, sendo de mais fácil tratamento e destinação final do que o resíduo mineral gerado pelo Sulfato de Alumínio e o Cloreto Férrico, com potencial de servir de insumo para a agricultura e até mesmo geração de biocombustíveis. Além das vantagens ambientais, existe a possibilidade de produção local dos coagulantes naturais (POUMAYE et al., 2012). 
A partir do que foi exposto, o presente estudo tem como objetivo a avaliação do desempenho de quatro coagulantes, sendo dois de natureza orgânica, Tanfloc SL e Tanfloc SG, e dois de caráter inorgânico, Sulfato de Alumínio e Cloreto Férrico, na remoção de turbidez e cor verdadeira de uma amostra de água coletada de um reservatório superficial. Também foi verificada a influência dos fatores dosagem dos coagulantes e velocidade de sedimentação nos resultados dos ensaios de tratabilidade.

\section{MATERIAIS E MÉTODOS}

\section{Área de estudo}

A água bruta para os ensaios de tratabilidade foi coletada do açude de Bodocongó (70 13’ 11" S e 35은 52 ' 31" W), situado no médio curso do rio Paraíba, a uma altitude de 548 metros, na cidade de Campina Grande/PB. O açude possui área da bacia hidráulica de $371.897 \mathrm{~m}^{2}$, capacidade e profundidade máxima de 1.020.000 $\mathrm{m}^{3}$ e $8,5 \mathrm{~m}$, respectivamente (MOREDJO, 1998). O mesmo está localizado na bacia do Riacho de Bodocongó, esta, por sua vez, abrange ao norte localidades externas aos limites urbanos de Campina Grande, como os municípios de Pocinhos e Puxinanã (TSUYUGUCHI, 2015). Na Figura 1 encontra-se a localização do açude de Bodocongó e sua bacia de contribuição.

A bacia do Riacho de Bodocongó está, de modo geral, em processo de urbanização, possuindo baixa impermeabilização nas sub-bacias a montante do açude e nas sub-bacias ao sul, além de apresentar subbacias com uma taxa de impermeabilização de 68\% (TSUYUGUCHI, 2015).

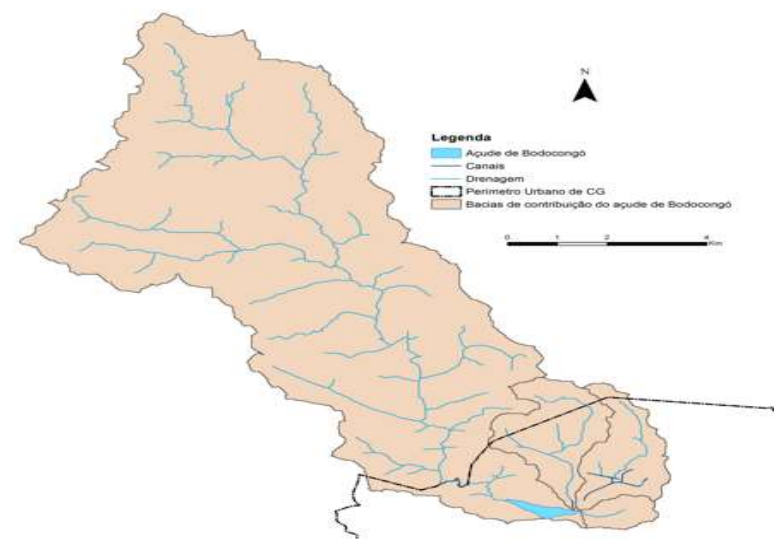

Figura 1: Mapa de localização da área de estudo.

\section{Coleta, armazenamento e monitoramento da água de estudo}

Os procedimentos para coleta, armazenamento e monitoramento da água de estudo foram efetuados de acordo com as recomendações preconizadas por Di Bernardo (2011). Foram coletados 1500 litros de água do açude Bodocongó, o volume foi armazenado em um reservatório de fibra de vidro de 2000 litros para posterior execução dos ensaios de tratabilidade. No decorrer dos ensaios foi realizado o monitoramento dos parâmetros físico-químicos: turbidez, cor verdadeira, $\mathrm{pH}$, alcalinidade e temperatura. Todos os métodos analíticos seguiram as recomendações do standard methods for the examination of water and Wastewater (APHA, 2012). 


\section{Produtos químicos e condições experimentais}

O Sulfato de Alumínio $\left(\mathrm{Al}_{2}(\mathrm{SO} 4)_{3} .16 \mathrm{H}_{2} \mathrm{O}\right)$, Cloreto Férrico $\left(\mathrm{FeCL}_{3} .8 \mathrm{H}_{2} \mathrm{O}\right)$, Tanfloc SL e Tanfloc SG foram utilizados como coagulantes primários para a realização da clarificação da água bruta de estudo. As soluções de todos os coagulantes foram preparadas a $1 \%\left(10 \mathrm{~g} . \mathrm{L}^{-1}\right)$. Neste trabalho, os coagulantes a base de tanino foram cedidos pela empresa TANAC ${ }^{\oplus}$.

Os ensaios de coagulação/floculação/decantação da água de estudo foram efetuados em um aparelho Jar test modelo MILAN JAR TEST-JT 102. As seguintes condições operacionais foram estabelecidas para os ensaios: para a mistura rápida foi utilizada a rotação de 300 rpm em um tempo de 20 segundos, o período de floculação ocorreu em 15 minutos, variando as rotações em 60, 40 e 30 rpm, respectivamente, a cada 5 minutos. A etapa de sedimentação foi baseada nas velocidades de sedimentação de 1,5 e 3,0 cm.min

1. As dosagens dos coagulantes variaram de 10 a $70 \mathrm{mg}$. $\mathrm{L}^{-1}$, com intervalos de $10 \mathrm{mg}$. L $\mathrm{L}^{-1}$. Para a obtenção de amostras que permitissem a aplicação de métodos estatísticos em consonância com a natureza dos dados obtidos, cada ensaio de tratabilidade foi realizado com 10 repetições.

\section{Verificação da influência da velocidade de sedimentação e da dosagem dos coagulantes}

Para comparar os valores de cada parâmetro obtidos para as duas velocidades de sedimentação, foi utilizado o teste T de Student para dados pareados. Já para a verificação da influência da dosagem, foi aplicado o teste de comparações múltiplas de Tukey. Antes da aplicação dos testes paramétricos foi necessário verificar se as amostras aderem a distribuição normal, uma vez que a normalidade dos dados é uma premissa para a aplicação de testes dessa natureza. Para isso, foi utilizado o teste de Shapiro-Wilk.

O software de análise estatística Action Stat, em conjunto com o Excel, foi utilizado para realizar todas as análises estatísticas propostas nesse estudo, considerando um nível de confiança de $95 \%$ (ou pvalor de 0,05$)$. Tanto o teste T de Student como o de Tukey foram realizados considerando uma hipótese nula de igualdade entre as amostras.

\section{RESULTADOS E DISCUSSÃO}

\section{Caracterização e monitoramento da água bruta}

Na Tabela 1 encontram-se os valores calculados das médias aritméticas, e seus respectivos desvios padrões, para os conjuntos de dados de cada parâmetro físico-químico monitorado. A partir dos resultados expressos na Tabela 1 verifica-se que foram obtidos valores de desvios padrão consideravelmente pequenos para os parâmetros monitorados, não apresentando alterações significativas em sua qualidade durante a realização dos procedimentos experimentais.

Tabela 1: Monitoramento da água de estudo.

\begin{tabular}{|l|l|}
\hline Parâmetros & Valores \\
\hline Temperatura & $27,45 \pm 0,52$ oC \\
\hline Turbidez & $22,11 \pm 1,58 \mathrm{uT}$ \\
\hline Cor verdadeira & $52,22 \pm 1,06 \mathrm{uH}$ \\
\hline Alcalinidade & $339,09 \pm 3,02 \mathrm{CaCO}_{3} \mathrm{mg} \mathrm{L}^{-1}$ \\
\hline $\mathrm{pH}$ & $8,14 \pm 0,06$ \\
\hline
\end{tabular}




\section{Resultados da determinação da turbidez e cor verdadeira para as amostras de água decantada}

Por meio da Figura 2 é possível verificar o desempenho na remoção de turbidez, para os quatro coagulantes utilizados, nas diferentes condições de sedimentação. Em relação ao Tanfloc SL, para a velocidade de sedimentação de $3,0 \mathrm{~cm} \cdot \mathrm{min}^{-1}$, é coerente afirmar que o mesmo foi superior aos dois coagulantes inorgânicos. Tem-se que a menor eficiência de remoção apresentada pelo Tanfloc SL, 90,40\%, foi mais efetiva do que o melhor índice de remoção proporcionado pelo Sulfato de Alumínio, 87,74\%. Quando a comparativa é realizada com o Cloreto Férrico, apenas a dosagem de $10 \mathrm{mg}$. $\mathrm{L}^{-1}$ do sal de ferro apresentou turbidez menor do que a mesma dosagem aplicada para o Tanfloc SL (1,96 uT para o Cloreto Férrico e 2,12 UT referente ao Tanfloc SL). Para as demais dosagens, o melhor desempenho foi obtido quando a água foi coagulada com o polímero orgânico.

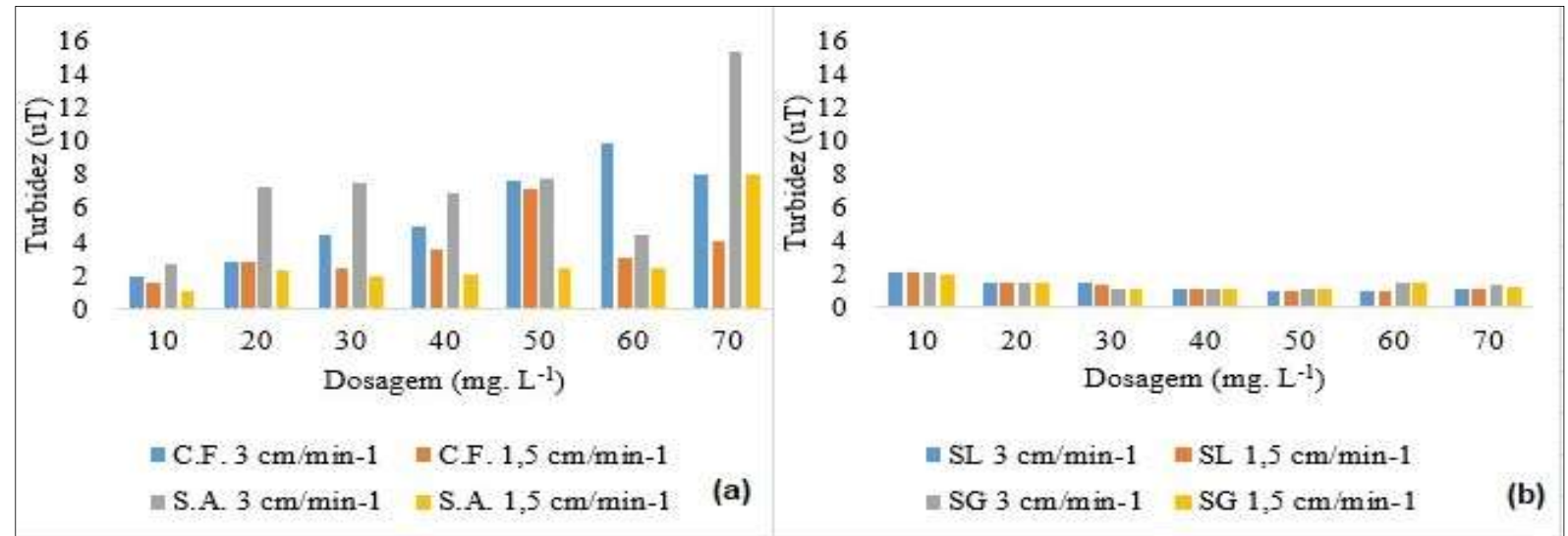

Figura 2: Valores de turbidez para os ensaios com Sulfato de Alumínio e Cloreto Férrico (a) e para o Tanfloc SL e Tanfloc SG (b). [C.F. = Cloreto Férrico, S.A. = Sulfato de Alumínio, SL = Tanfloc SL e SG = Tanfloc SG].

A Portaria de $n^{\circ} 5$ de 2017, que dispõe sobre a consolidação das normas sobre as ações e os serviços de saúde do Sistema Único de Saúde, estabelece que na saída das estações de tratamento de água os valores de turbidez não devem ser superiores a 0,5 UT em ao menos $95 \%$ das amostras mensais. Tomando como base o valor da Portaria de consolidação $\mathrm{n}^{\circ} 5 / 2017$, as dosagens de $50 \mathrm{mg}^{\mathrm{L}} \mathrm{L}^{-1}$ e $60 \mathrm{mg}$. $\mathrm{L}^{-1}$ de Tanfloc SL, nas duas velocidades de sedimentação estudadas, proporcionaram uma produção de água decantada com níveis de turbidez próximos ao exigido pela legislação (0,92 UT e 0,96 UT para $50 \mathrm{mg}$. L-1 e $60 \mathrm{mg}$. L-1, ambos correspondentes a velocidade de sedimentação de $1,5 \mathrm{~cm} \cdot \mathrm{min}^{-1}$ ), ressaltando que a água decantada ainda seria submetida a etapa de filtração, sendo possível atender aos níveis de turbidez estabelecidos pela respectiva portaria. O Sulfato de Alumínio e o Cloreto Férrico não apresentaram reduções na turbidez como as discutidas anteriormente.

Maiores índices de remoção de turbidez podem refletir em uma melhoria da qualidade microbiológica da água. A utilização da turbidez como indicador da eficiência do tratamento de água encontra duas aplicações principais, a primeira envolve a remoção de cistos e oocistos de protozoários por meio de filtração e a segunda, do bom condicionamento da água para a pré-desinfecção, já que partículas suspensas podem proteger os Microrganismos dos efeitos dos agentes desinfetantes (WHO, 2004). 
O desempenho do Tanfloc SG foi similar ao apresentado pelo Tanfloc SL, porém com melhor remoção de turbidez correspondente a 95,11\%, resultando em uma turbidez remanescente, na água decantada, de 1,08 UT. O desempenho mais efetivo do Tanfloc SG diz respeito a dosagem de $30 \mathrm{mg}^{-1} \mathrm{~L}^{-1}$ e velocidade de sedimentação correspondente a $1,5 \mathrm{~cm} \cdot \mathrm{min}^{-}$

As menores dosagens de Tanfloc SL e Tanfloc SG também foram responsáveis por uma eficiência significativa na remoção dos níveis de turbidez da água bruta (Figura 2), algo que está em consonância com trabalhos desenvolvidos por Beltran-Heredia et al. (2009), tais autores destacam que para a remoção de material em suspensão, reduzidas dosagens de coagulantes a base de tanino são suficientes para a obtenção de eficiência elevada. Desta forma, a possibilidade de se trabalhar com menores dosagens de coagulantes permite uma redução nos custos do tratamento e uma menor geração de resíduos.

Avaliando os custos da inserção do Tanfloc SL nas unidades de tratamento de água para abastecimento público, Rôla et al. (2016) concluíram que este coagulante permite uma redução global dos custos das etapas de coagulação/floculação e cloração de até $49,61 \%$, se comparados, por exemplo, a coagulantes como o PAC (Policloreto de Alumínio).

A melhor performance dos coagulantes a base de tanino pode estar atrelada ao mecanismo de coagulação predominante a partir da adição desses produtos na etapa de mistura rápida. Segundo Libânio (2010), os polímeros apresentam longas cadeias formadas por monômeros, que podem ter seus sítios carregados positivamente, desta forma, os flocos formados pelo Tanfloc possuem maior área superficial do que os associados ao Sulfato de Alumínio e ao Cloreto Férrico, com elevado peso molecular e, consequentemente, maior velocidade de sedimentação.

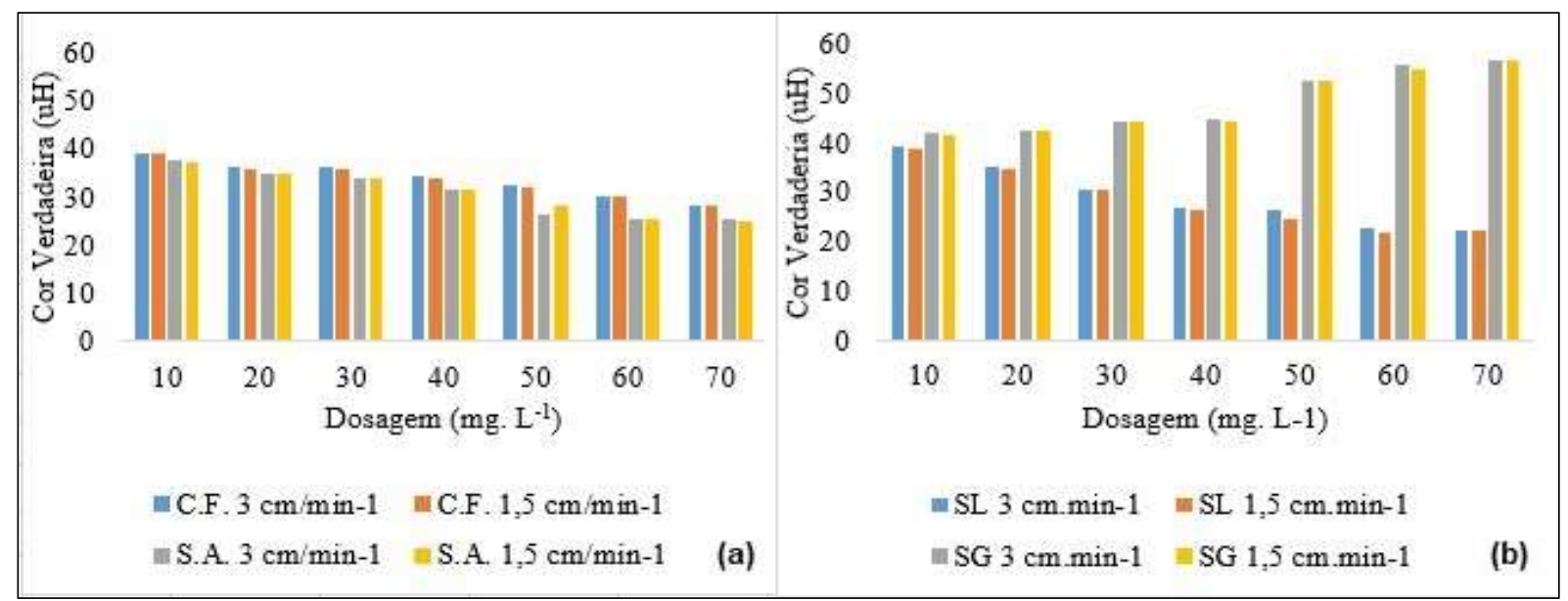

Figura 3: Valores de cor verdadeira para os ensaios com o Sulfato de Alumínio e Cloreto Férrico (a) e para o Tanfloc SL e Tanfloc SG (b). [C.F. = Cloreto Férrico, S.A. = Sulfato de Alumínio, SL = Tanfloc SL e SG = Tanfloc SG].

Em estudo comparativo entre o Tanfloc SL, Tanfloc SG e o Sulfato de Alumínio, Piantá (2008) pôde observar que durante os ensaios de tratabilidade, os coagulantes a base de tanino proporcionaram a formação de flocos maiores e, aparentemente, com massa específica superior aos encontrados quando a água foi coagulada com o sal de alumínio. Yin (2010) ainda ressalta que quanto mais longas, as cadeias poliméricas apresentam um maior número de sítios de adsorção livres, o que propicia a agregação de uma 
maior quantidade de impurezas. O desempenho dos coagulantes em relação a cor verdadeira para as amostras de água decantada pode ser visualizado na Figura 3.

Conforme verificado na Figura 3.a, a atuação do Cloreto Férrico na remoção da cor verdadeira foi similar ao desempenho apresentado pelo Sulfato de Alumínio, com baixos percentuais de remoção. Quando estudada a velocidade de sedimentação de $1,5 \mathrm{~cm}$. $\mathrm{min}^{-1}$ e dosagem de $70 \mathrm{mg} \cdot \mathrm{L}^{-1}$, tanto o Sulfato de Alumínio como o Cloreto Férrico apresentaram maior índice de remoção, 51,92\% e 46\%, respectivamente.

Os índices mais discretos de remoção de cor verdadeira, em relação aos encontrados para turbidez, podem ser justificados com base na natureza das partículas responsáveis pela coloração nas águas. Conforme mencionado por Di Bernardo (2011), a cor verdadeira nas águas superficiais ocorre, geralmente, devido à presença de matéria orgânica dissolvida ou de ferro. Desta forma, tais partículas apresentam menores dimensões do que as suspensas, com menor densidade e, consequentemente, menor velocidade de sedimentação. Essa dificuldade é comumente encontrada em ETA's no qual o tratamento é direcionado a águas com teores de cor verdadeira acentuados (JULIO et al., 2006), sendo necessária a aplicação de auxiliares de floculação ou, como na maioria dos casos, a oxidação dessa matéria orgânica (WANG et al., 2008), o que pode tornar o tratamento mais oneroso, além de acarretar a formação de subprodutos orgânicos halogenados (SOH) nocivos à saúde, tais como trihalometanos (DANTAS et al., 2011).

Julio et al. (2006) evidenciam que no tratamento de águas que possuem baixa turbidez e elevada cor, existe uma redução da probabilidade de ocorrência de choques entre as partículas, o que dificulta sua agregação e, consequentemente, a formação dos flocos, reduzindo a eficiência da operação de floculação. A eficiência de remoção da cor verdadeira por parte do Tanfloc SL foi mais elevada para a dosagem de $10 \mathrm{mg}$. $\mathrm{L}^{-1}$, com redução de 19,54 \% e 19,96\%, para Vs $=3 \mathrm{~cm} \cdot \mathrm{min}^{-1}$ e Vs $=1,5 \mathrm{~cm} \cdot \mathrm{min}^{-1}$, respectivamente, enfatizando que o valor médio apresentado pela água de estudo para a cor verdadeira foi de 52,2 uH. Em relação ao Tanfloc SG, em dosagens superiores a $50 \mathrm{mg}$. $\mathrm{L}^{-1}$ não ocorreu remoção de cor verdadeira, e sim um acréscimo ao valor médio apresentado pela água bruta.

\section{Resultados da aplicação do teste de shapiro-wilk}

Na Tabela 2 encontram-se os resultados da aplicação do teste Shapiro-Wilk para as amostras correspondentes aos resultados de turbidez e cor verdadeira para a água decantada que foi coagulada com o Sulfato de Alumínio e o Cloreto Férrico. Verifica-se que todas as amostras submetidas ao teste de normalidade aderem a distribuição normal. Todos os resultados determinados para os $p$-valores foram maiores do que o $p$-valor adotado, desta forma, as amostras provem de uma população normal. Assim, para esses resultados, a aplicação dos testes paramétricos sugeridos torna-se possível. Sob as mesmas condições da Tabela 2, foi elaborada a Tabela 3, porém com os resultados do teste de normalidade para as amostras associadas aos coagulantes orgânicos.

De maneira similar aos resultados obtidos para o Sulfato de Alumínio e o Cloreto Férrico, as amostras referentes aos parâmetros encontrados para a água decantada que foi coagulada com o Tanfloc SL e o Tanfloc SG também mostraram aderência a distribuição normal. 
Tabela 2: Resultados da aplicação do teste Shapiro-Wilk para as amostras dos coagulantes inorgânicos.

\begin{tabular}{|l|l|l|l|l|l|l|l|l|l|}
\hline \multirow{2}{*}{$\begin{array}{l}\text { Dosagem (mg. } \\
\left.\mathrm{L}^{-1}\right)\end{array}$} & $\begin{array}{l}\text { Cloreto Férrico } \\
\text { Turbidez }\end{array}$ & $\begin{array}{l}\text { Cloreto Férrico Cor } \\
\text { Verdadeira }\end{array}$ & $\begin{array}{l}\text { Sulfato de Alumínio } \\
\text { Turbidez }\end{array}$ & $\begin{array}{l}\text { Sulfato de Alumínio Cor } \\
\text { Verdadeira }\end{array}$ \\
\cline { 2 - 10 } & Velocidade de Sedimentação $\left(\mathrm{cm} . \mathrm{min}^{-1}\right)$ \\
\cline { 2 - 10 } & 3,0 & 1,5 & 3,0 & 1,5 & 3,0 & 1,5 & 3,0 & 1,5 \\
\hline 10 & 0,3915 & 0,0632 & 0,8657 & 0,6592 & 0,4391 & 0,1629 & 0,7578 & 0,1421 \\
\hline 20 & 0,4155 & 0,0845 & 0,8140 & 0,7471 & 0,6406 & 0,8764 & 0,8140 & 0,9776 \\
\hline 30 & 0,7242 & 0,6976 & 0,4458 & 0,5527 & 0,6947 & 0,5207 & 0,9249 & 0,9573 \\
\hline 40 & 0,2446 & 0,3290 & 0,8580 & 0,8457 & 0,5471 & 0,2350 & 0,0582 & 0,0844 \\
\hline 50 & 0,4101 & 0,8445 & 0,4030 & 0,0729 & 0,5218 & 0,2840 & 0,7296 & 0,0669 \\
\hline 60 & 0,4924 & 0,6176 & 0,2515 & 0,4689 & 0,0705 & 0,1716 & 0,5282 & 0,0563 \\
\hline 70 & 0,7393 & 0,0868 & 0,8102 & 0,8129 & 0,4919 & 0,0525 & 0,3331 & 0,7456 \\
\hline
\end{tabular}

Tabela 3: Resultados da aplicação do teste Shapiro-Wilk para as amostras dos coagulantes orgânicos.

\begin{tabular}{|c|c|c|c|c|c|c|c|c|}
\hline \multirow[t]{3}{*}{ Dosagem (mg. $\mathrm{L}^{-1}$ ) } & \multicolumn{2}{|c|}{ Tanfloc SL - Turbidez } & \multicolumn{2}{|c|}{ Tanfloc SL - Cor Verdadeira } & \multicolumn{2}{|c|}{ Tanfloc SG - Turbidez } & \multicolumn{2}{|c|}{ Tanfloc SG - Cor Verdadeira } \\
\hline & \multicolumn{8}{|c|}{ Velocidade de Sedimentação (cm. min-1) } \\
\hline & 3,0 & 1,5 & 3,0 & 1,5 & 3,0 & 1,5 & 3,0 & 1,5 \\
\hline 10 & 0,2384 & 0,5434 & 0,3819 & 0,9546 & 0,0860 & 0,7126 & 0,8542 & 0,3936 \\
\hline 20 & 0,4510 & 0,8157 & 0,0675 & 0,0752 & 0,3548 & 0,2776 & 0,0630 & 0,7091 \\
\hline 30 & 0,1892 & 0,0959 & 0,6446 & 0,9697 & 0,2475 & 0,0606 & 0,7316 & 0,7347 \\
\hline 40 & 0,5097 & 0,3603 & 0,0895 & 0,1510 & 0,1199 & 0,1639 & 0,2281 & 0,0708 \\
\hline 50 & 0,8989 & 0,4976 & 0,7625 & 0,3317 & 0,9898 & 0,5829 & 0,5435 & 0,5702 \\
\hline 60 & 0,2691 & 0,9937 & 0,7941 & 0,9610 & 0,6294 & 0,6264 & 0,2716 & 0,2932 \\
\hline 70 & 0,4099 & 0,1716 & 0,0801 & 0,6392 & 0,5523 & 0,2851 & 0,0686 & 0,0860 \\
\hline
\end{tabular}

\section{Influência da velocidade de sedimentação}

Os resultados da aplicação do teste T de Student para todas as mostras de dados relacionados a água decantada a partir da coagulação com o Cloreto Férrico e com o Sulfato de Alumínio estão expressos na Tabela 4. Os resultados para cada $p$-valor abaixo do nível de significância $(0,05)$ estão destacados em negrito.

Tabela 4: Resultados do teste T de Student para os coagulantes inorgânicos.

\begin{tabular}{|c|c|c|c|c|c|}
\hline \multirow{2}{*}{$\begin{array}{l}\begin{array}{l}\text { Dosagem } \\
\left(\mathrm{mg}^{-1} \text {. }\right.\end{array} \\
\end{array}$} & \multirow[t]{2}{*}{ Análise Estatística } & \multicolumn{2}{|c|}{ Parâmetros Analisados - Sulfato de Alumínio } & \multicolumn{2}{|c|}{ Parâmetros Analisados - Cloreto Férrico } \\
\hline & & Turbidez & Cor Verdadeira & Turbidez & Cor Verdadeira \\
\hline & & $2,05 E-05$ & $2,03 \mathrm{E}-01$ & 6,471E-02 & 0,1534 \\
\hline & & $2,89 \mathrm{E}-07$ & $7,77 \mathrm{E}-02$ & 7,801E-02 & 0,3046 \\
\hline & & $8,08 E-07$ & $2,96 \mathrm{E}-01$ & 3,939E-03 & 0,1419 \\
\hline 40 & P-valor & $1,84 \mathrm{E}-07$ & $1,08 \mathrm{E}-01$ & 2,993E-06 & 0,2501 \\
\hline 50 & P-valor & $1,02 E-08$ & $4,19 E-01$ & 1,614E-03 & 0,3380 \\
\hline 60 & P-valor & $2,00 E-07$ & 7,51E-02 & 1,501E-07 & 0,7955 \\
\hline 70 & P-valor & 1,31E-06 & $2,47 E-01$ & $3,450 E-08$ & 0,1951 \\
\hline
\end{tabular}

O Sulfato de Alumínio apresentou uma diferença, estatisticamente significativa, para os valores de turbidez encontrados, para as duas velocidades de sedimentação, enquanto o Cloreto Férrico não possuiu tal dependência da velocidade apenas nas dosagens de 10 e $20 \mathrm{mg}$. $\mathrm{L}^{-1}$. Desta forma, após o período de sedimentação associado a $3,0 \mathrm{~cm} \cdot \mathrm{min}^{-1}$, uma quantidade significativa de sólidos suspensos ainda permaneceu na água decantada. Em estudo que objetivou a obtenção de diagramas de coagulação para o Cloreto Férrico e o Sulfato de Alumínio, Bartiko et al. (2015) observaram que, para esses coagulantes, as amostras de água decantada associadas a menores velocidades de sedimentação apresentaram qualidade significativamente superior, em termos de turbidez, o que corrobora com os resultados expostos na Tabela 4. 
Conforme destacado por Pádua et al. (2002), os flocos formados pelo Cloreto Férrico possuem maior densidade do que os produzidos com o Sulfato de Alumínio, apresentando, desta forma, maior velocidade de sedimentação. Assim, para as dosagens de 10 e $20 \mathrm{mg}$. $\mathrm{L}^{-1}$ de Cloreto Férrico, durante o período de sedimentação associado a velocidade de $3,0 \mathrm{~cm} \cdot \mathrm{min}^{-1}$, a maioria dos flocos já haviam sedimentado, permanecendo uma parcela ínfima que sedimentou quando fixado o tempo de sedimentação de acordo com 1,5 cm. min ${ }^{-1}$. É importante comentar que, em termos de $\mathrm{pH}$, o Sulfato de Alumínio pôde atuar dentro de uma faixa ótima para esse coagulante. Segundo Rosalino (2011), o Sulfato de Alumínio é mais eficiente em valores de $\mathrm{pH}$ compreendidos entre 5,5 e 8,5.

Ainda analisando os resultados da Tabela 4, é possível visualizar que diferentemente do que ocorreu com o parâmetro turbidez, os valores encontrados para a cor verdadeira não apresentaram dependência da velocidade de sedimentação, sendo, portanto, estatisticamente iguais para as velocidades de sedimentação de 3 e $1,5 \mathrm{~cm} \cdot \mathrm{min}^{-1}$, em todas as dosagens analisadas. A independência para a cor verdadeira pode ser justificada pelo fato de que as partículas responsáveis por conferir os níveis desse parâmetro a água possuem pequenas dimensões e baixa densidade, apresentando, consequentemente, baixas velocidades de sedimentação.

Ao se proporcionar maiores tempos de sedimentação, uma melhoria na qualidade da água decantada pode ser observada, principalmente no caso do Sulfato Alumínio aqui apresentado, porém, todas as respostas a essas medidas devem ser avaliadas. Unidades de decantação dimensionadas a partir de baixas taxas de sedimentação demandam grandes áreas para sua implantação, devido ao maior tempo de detenção hidráulica, o que pode tornar o projeto de uma ETA inviável do ponto de vista econômico (BARTIKO et al., 2015). Por outro lado, uma melhor qualidade para a água decantada é responsável por uma operação mais efetiva das unidades de filtração, que pode ocorrer com maior tempo de carreira (MACHADO et al., 2011). $\mathrm{Na}$ Tabela 5 estão presentes os resultados da aplicação do teste T de Student para os coagulantes orgânicos, com os mesmos critérios de apresentação de cada $p$-valor fixados para os resultados referentes ao Sulfato de Alumínio e ao Cloreto Férrico (Tabela 4).

Tabela 5: Resultados do teste T de Student para os coagulantes orgânicos.

\begin{tabular}{|c|c|c|c|c|c|}
\hline Dosagem (mg. L $^{-1}$ ) & Análise Estatística & \multicolumn{2}{|c|}{ Parâmetros Analisados - Tanfloc SL } & \multicolumn{2}{c|}{ Parâmetros Analisados - Tanfloc SG } \\
\cline { 3 - 5 } & & Turbidez & Cor Verdadeira & Turbidez & Cor Verdadeira \\
\hline 10 & P-valor & 0,0533 & 0,1452 & 0,1045 & 0,3940 \\
\hline 20 & P-valor & 0,0972 & 0,0777 & 0,1193 & 0,2262 \\
\hline 30 & P-valor & 0,2080 & 0,1138 & 0,1087 & 0,2663 \\
\hline 40 & P-valor & 0,0608 & 0,0890 & 0,1180 & 0,0533 \\
\hline 50 & P-valor & 0,0941 & 0,2360 & 0,1538 & 0,0993 \\
\hline 60 & P-valor & 0,1452 & 0,1210 & 0,1404 & 0,3670 \\
\hline 70 & P-valor & 0,1707 & 0,1641 & 0,1803 & 0,5740 \\
\hline
\end{tabular}

Os resultados demonstram que tanto o Tanfloc SL como o Tanfloc SG não apresentaram, em nenhuma das dosagens, uma dependência significativa da velocidade de sedimentação, para esses coagulantes, os valores de turbidez e cor verdadeira são estatisticamente semelhantes para as duas velocidades estudadas. 
A possibilidade fornecida pelos coagulantes orgânicos de se trabalhar com maiores velocidades de sedimentação advém da elevada capacidade que estes coagulantes possuem de formar flocos mais densos e com uma velocidade de sedimentação superior aos formados pelo Sulfato de Alumínio e Cloreto Férrico (DI BERNARDO et al., 2005). Assim, os flocos originados pela adição do Tanfloc, caracterizados por longas cadeias poliméricas, permitiram que um grande número de impurezas presentes na água pudesse ser agregadas, culminando em uma água decantada de melhor qualidade, com menores valores de cor e turbidez.

Santos et al. (2004) ressaltam que o principal parâmetro de projeto de unidades de sedimentação é a taxa de aplicação superficial. De um ponto de vista prático, com a implantação de unidades de sedimentação/decantação correspondentes a uma velocidade de sedimentação de $3 \mathrm{~cm} \cdot \mathrm{min}^{-1}$, economias significativas de recursos financeiros, materiais e humanos podem ser obtidos, se comparados ao dimensionamento para uma velocidade de sedimentação inferior, no caso de $1,5 \mathrm{~cm}$. $\mathrm{min}^{-1}$, já que, segundo Cavazzana et al. (2008), em sistemas de tratamento de água do tipo convencional, os custos das unidades de decantação e floculação podem ser responsáveis por 75\% da área total de toda ETA.

\section{Verificação da influência da dosagem}

A aplicação do teste de Tukey tomou como base os resultados do teste T de Student, quando não houve dependência do fator velocidade de sedimentação, o teste foi realizado apenas para os resultados correspondentes a velocidade de 3,0 cm. $\mathrm{min}^{-1}$, e, quando ocorreu tal dependência, o teste de Tukey foi aplicado as amostras pertencentes as duas velocidades de sedimentação. A Tabela 6 demonstra os resultados das análises de variância unifatorial (ANOVA) para os parâmetros referentes ao Cloreto Férrico e ao Sulfato de Alumínio. O p-valor em negrito indica que o fator dosagem dos coagulantes foi relevante para os valores de turbidez e cor verdadeira da água decantada.

Tabela 6: ANOVA unifatorial para os coagulantes inorgânicos.

\begin{tabular}{|c|c|c|c|}
\hline Coagulantes & Parâmetros & Velocidade de Sedimentação & P-valor \\
\hline Cloreto Férrico & Turbidez & $3,0 \mathrm{~cm} \cdot \mathrm{min}-1$ & $\mathbf{1 , 0 7 2 5 \mathrm { E } - \mathbf { 2 8 }}$ \\
\cline { 3 - 4 } & & $1,5 \mathrm{~cm} \cdot \mathrm{min}-1$ & $\mathbf{5 , 6 2 8 6 \mathrm { E } - 2 2}$ \\
\hline Cloreto Férrico & Cor Verdadeira & $3,0 \mathrm{~cm} \cdot \mathrm{min}-1$ & $\mathbf{4 , 8 1 4 6 \mathrm { E } - 1 8}$ \\
\hline Sulfato de Alumínio & Turbidez & $3,0 \mathrm{~cm} \cdot \mathrm{min}-1$ & $\mathbf{8 , 1 9 5 3 \mathrm { E } - 3 4}$ \\
\cline { 3 - 4 } & & $1,5 \mathrm{~cm} \cdot \mathrm{min}-1$ & $\mathbf{8 , 3 5 0 4 \mathrm { E } - 3 9}$ \\
\hline Sulfato de Alumínio & Cor Verdadeira & $3,0 \mathrm{~cm} \cdot \mathrm{min}-1$ & $\mathbf{3 , 0 0 3 3 E - 2 2}$ \\
\hline
\end{tabular}

Os resultados de todos os parâmetros determinados para a água decantada, correspondentes tanto ao Sulfato de Alumínio como ao Cloreto Férrico, tiveram influência significativa da dosagem dos respectivos coagulantes. Desta forma, ao menos duas amostras, para cada teste realizado apresentaram divergência quando comparadas, sendo possível identificar a partir do teste de Tukey onde estão as diferenças responsáveis por tais resultados. A Figura 4 apresenta o resultado do Teste de Tukey os dados de turbidez associados ao Cloreto Férrico. 


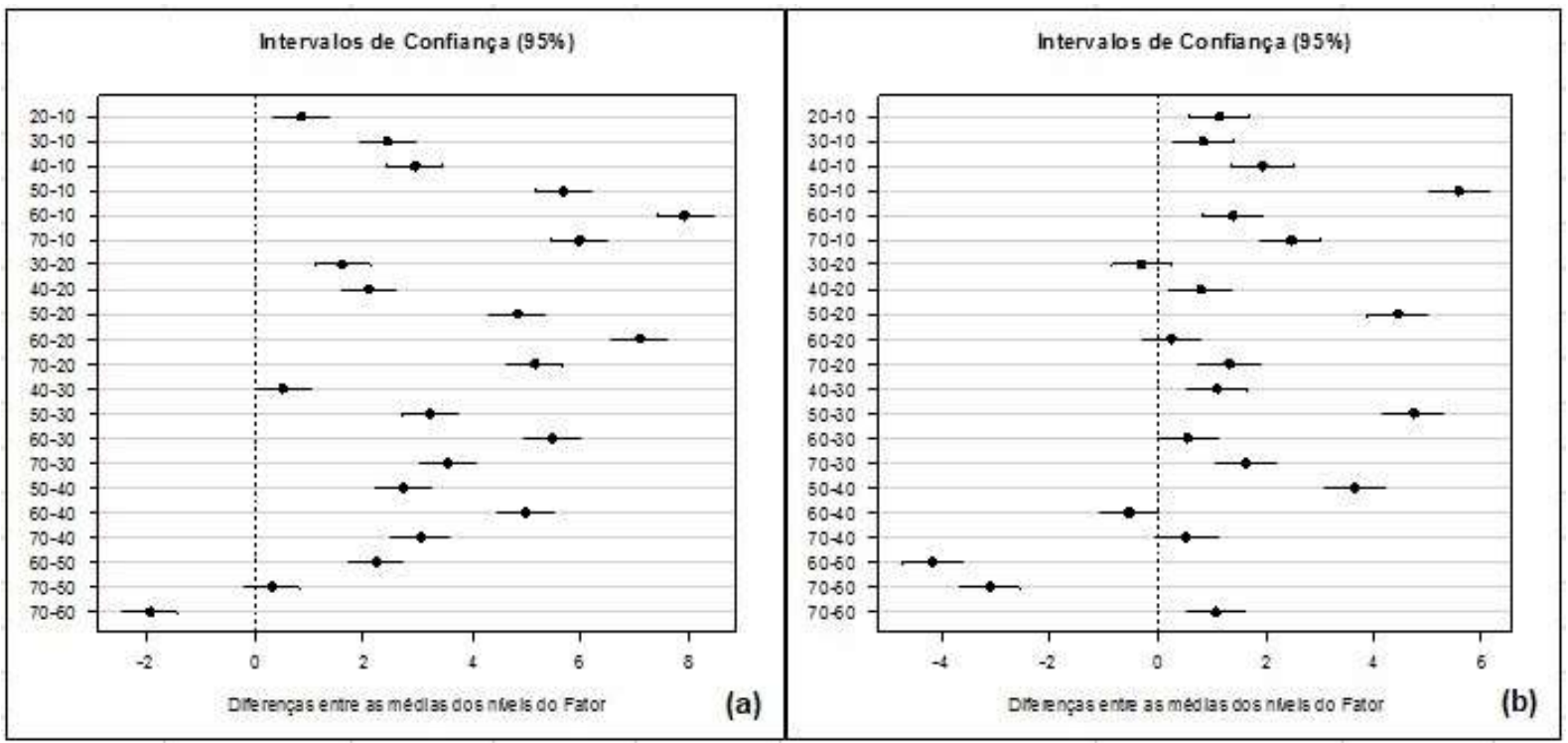

Figura 4: Teste de Tukey para os valores de turbidez relacionados ao Cloreto Férrico com velocidade de sedimentação de $3,0 \mathrm{~cm} \cdot \mathrm{min}^{-1}$ (a) e $1,5 \mathrm{~cm} \cdot \mathrm{min}^{-1}$ (b).

Com base na Figura 4, é possível observar que a influência da dosagem ocorreu de maneira distinta para as duas velocidades de sedimentação, embora o mesmo coagulante e dosagens estejam sendo levadas em questão. O fato argumentado na assertiva anterior reforça a significativa influência da própria velocidade de sedimentação na dinâmica do tratamento realizado com o Cloreto Férrico. Como a água decantada apresentou distinção, em termos de turbidez, consequentemente, a influência da dosagem ocorreu de maneiras distintas nas respectivas amostras. Na Tabela 7 encontram-se os resultados das análises de variância unifatoriais (ANOVA) para todos os parâmetros referentes ao uso do Tanfloc SL e ao Tanfloc SG.

Tabela 7: ANOVA unifatorial para os coagulantes orgânicos.

\begin{tabular}{|c|c|c|c|}
\hline Coagulantes & Parâmetros & Velocidade de Sedimentação & P-valor \\
\hline Tanfloc SL & Turbidez & $3,0 \mathrm{~cm} \cdot \mathrm{min}-1$ & $7,8088 \mathrm{E}-11$ \\
\hline Tanfloc SL & Cor Verdadeira & $3,0 \mathrm{~cm} \cdot \mathrm{min}-1$ & $5,1857 \mathrm{E}-12$ \\
\hline Tanfloc SG & Turbidez & $3,0 \mathrm{~cm} \cdot \mathrm{min}-1$ & $4,0842 \mathrm{E}-03$ \\
\hline Tanfloc SG & Cor Verdadeira & $3,0 \mathrm{~cm} \cdot \mathrm{min}-1$ & $8,3667 E-14$ \\
\hline
\end{tabular}

Por meio da Tabela 7 é verificado que a dosagem dos coagulantes a base de tanino teve influência significativa nos resultados determinados para turbidez e cor verdadeira da água decantada, sendo possível a aplicar o teste de Tukey. Para o Tanfloc SG e o Tanfloc SL, após a aplicação do teste de Tukey destacam-se os resultados referentes ao parâmetro turbidez, com repostas ao método estatístico similares para ambos os coagulantes (Figura 5).

Por meio da Figura 5.b é possível observar que a partir da adição de $40 \mathrm{mg}$. $\mathrm{L}^{-1}$ de Tanfloc SL não ocorreu diferença significativa entre os valores de turbidez da água decantada. Esse resultado foi similar ao encontrado por Coral et al. (2009), que também verificou que dosagens mais elevadas de Tanfloc, superiores a $40 \mathrm{mg} \mathrm{L}^{-1}$ não resultam em percentuais de remoção de turbidez mais representativos. Sendo assim, é possível a aplicação de $40 \mathrm{mg}$. $\mathrm{L}^{-1}$ de maneira a obter percentuais de remoção de turbidez satisfatórios, uma vez que dosagens mais elevadas acarretam apenas em maiores gastos com coagulante, além da geração de mais lodo, sem a produção de água clarificada com qualidade superior. 

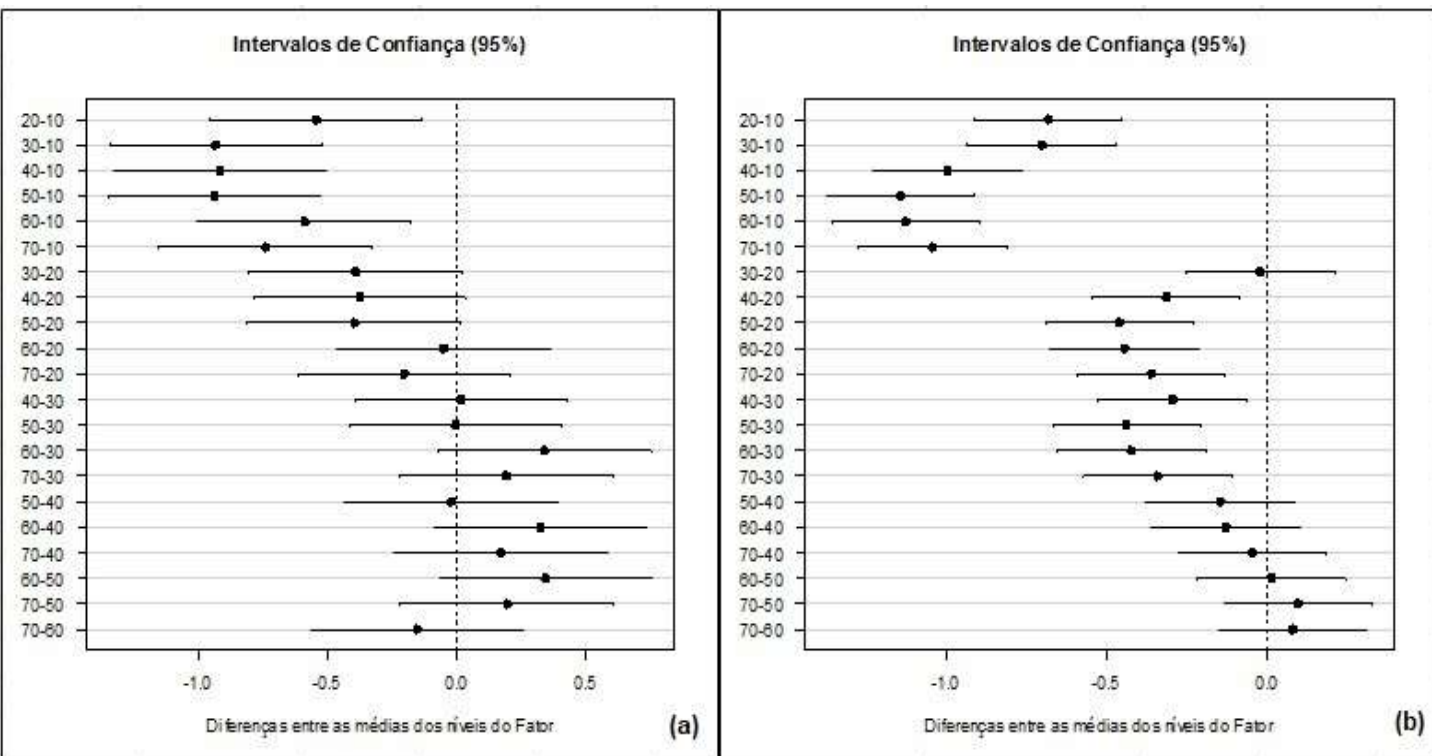

Figura 5: Teste de Tukey para os valores de turbidez relacionados ao Tanfloc SG (a) e ao Tanfloc SL (b).

A Figura $5 . a$ mostra que entre as dosagens $20 \mathrm{mg}^{\mathrm{L}^{-1}}$ e $70 \mathrm{mg} \cdot \mathrm{L}^{-1}$ de Tanfloc SG, diferenças significativas também não foram encontradas, quanto ao parâmetro turbidez. Desta forma, a aplicação de 20 mg. $L^{-1}$ para a remoção de turbidez da água em questão, pode proporcionar economias significativas, em termos de compra de coagulante e geração de lodo, sem comprometer a qualidade da água produzida. Avaliando o desempenho do Tanfloc SG na remoção de turbidez, visando usos para produção de águas potáveis, Gomes et al. (2017) destacam que para a remoção de turbidez, o respectivo coagulante se mostrou mais eficiente quando menores dosagens foram empregadas, obtendo-se elevados índices de remoção de material em suspensão utilizando-se menores dosagens do Tanfloc SG.

\section{CONCLUSÕES}

O coagulante orgânico Tanfloc SL se mostrou mais eficiente na remoção de turbidez e cor verdadeira, com os melhores índices de remoção desses parâmetros. Já o coagulante orgânico. A partir da aplicação do teste T de Student, nenhum dos coagulantes a base de tanino apresentaram dependência do fator velocidade de sedimentação, com valores de turbidez e cor verdadeira estatisticamente iguais para as duas velocidades de sedimentação trabalhadas. Desta forma, a fixação de uma maior velocidade de sedimentação, $3 \mathrm{~cm} . \mathrm{min}^{-}$ 1, acarreta em economias expressivas em termos de instalação e operação das unidades de decantação de uma ETA. Tanto o Sulfato de Alumínio como o Cloreto Férrico apresentaram dependência do fator velocidade de sedimentação, sendo necessário permitir maiores períodos de sedimentação para se obter uma água decantada de melhor qualidade.

A dosagem dos coagulantes foi um fator relevante para os resultados de todos os parâmetros submetidos ao teste de Tukey. Em especial, os coagulantes orgânicos demonstraram uma particularidade, com valores de turbidez remanescente iguais a partir de uma determinada dosagem. Assim, é possível realizar o tratamento com uma menor dosagem aplicada, gerando um menor custo com produtos químicos além de menor produção de lodo. 


\section{REFERÊNCIAS}

APHA. American Public Health Association. Standard methods for the examination of water and wastewater, 22nd ed.: American Water Works Association, Water Environment Federation. Washington: APHA, 2012.

BARTIKO, D.; JULIO, M. D.. Construção e emprego de diagramas de coagulação como ferramenta para o monitoramento contínuo da floculação em águas de abastecimento. Ambiente \& Agua - An Interdisciplinary Journal of Applied Science, v.10, n.1, 2015. DOI: http://dx.doi.org/10.4136/ambi-agua.1239

BRASIL. Ministério da Saúde. Portaria de consolidação № 05/2017: Dispõe sobre Consolidação das normas sobre as ações e os serviços de saúde do Sistema Único de Saúde. Diário Oficial da União, 2011.

BELTRÁN HEREDIA, J.; SÁNCHEZ MARTÍN, J.. Removing heavy metals from polluted surface water with a tannin-based flocculant agent. Journal of Hazardous Materials, v.165, n.13, p.1215-1218, 2009. DOI: http://dx.doi.org/10.1016/j.jenvman.2016.10.033

CAVAZZANA, T. L.; MATSUMOTO, T.; LIBÂNIO, M.. Avaliação da floco-decantação de manta de lodo associada à decantação de alta taxa no tratamento de águas de consumo humano. Engenharia Sanitaria e Ambiental, v.13, n.2, p.126-133, 2008

DOI: http://dx.doi.org/10.1590/S1413-41522008000200002

CORAL, L. A.; BERGAMASCO, R.; BASSETTI, F. J.. Estudo da Viabilidade de Utilização do Polímero Natural (TANFLOC) em Substituição ao Sulfato de Alumínio no Tratamento de Águas para Abastecimento Público. In: INTERNATION WORKSHOP ADVANCES IN CLEANER PRODUCTION, 2. Anais. São Paulo, 2009.

CORAL, L. A.. Remoção de cianobactérias e cianotoxinas em águas de abastecimento pela associação de flotação por ar dissolvido e nanofiltração. Dissertação (Mestrado em Engenharia Ambiental) - Universidade Federal de Santa Catarina, Florianópolis, 2009.

DANTAS, A. D. B.; PASCHOALATO, C. F. P. R.; FIGUEIREDO, J. O.; RAMOS, M. H. C.. Remoção de cor, de ferro e de manganês em água com matéria orgânica dissolvida por meio da pré-oxidação, coagulação, filtração e adsorção em carvão ativado granular. Revista DAE, v.59, n.186, p.46-55, 2011. DOI: http://dx.doi.org/10.4322/dae.2014.076

DI BERNARDO, L.; DANTAS, A. D. B.; VOLTAN, P. E. N. Tratabilidade de água e dos resíduos gerados em estações de tratamento de água. São Carlos: LDiBe Ltda, 2011.

FRANCO, C. S.; BRATISTA, M. D. A.; OLIVEIRA, L. F. C.; KOHN, G. P.; FIA. R.. Coagulação com semente de moringa oleifera preparada por diferentes métodos em águas com turbidez de 20 a 100 UNT. Revista Brasileira de Engenharia Sanitária e Ambiental, v.22, n.4, p.781-788, 2017. DOI: http://dx.doi.org/10.1590/S1413-41522017145729

GOMES, T. V. B.. Treatability of Surface Water Using Nature Coagulants Based on Tannin and Extract of Seeds. Ensaios e Ciência: Biológicas, Agrárias e da Saúde, v.21, p.152-155, 2017. DOI: http://dx.doi.org/10.17921/1415-

\subsection{7v21n3p152-155}

JULIO, M.; NEVES, E. F. A.; TROFINO, J. C.; DE BERNARDO, L.. Emprego do reagente de fenton como agente coagulante na remoção de substâncias húmicas de água por meio da flotação por ar dissolvido e filtração. Engenharia Sanitaria e Ambiental, v.11, n.3, p.260-268, 2006. DOI: http://dx.doi.org/10.1590/S1413-41522006000300009

LIBÂNIO, M.. Fundamentos de Qualidade e Tratamento de Água. 3 ed. Campinas: Átomo, 2010.

MACHADO, B. C.; SENS, M. L.. Remoção de cianobactérias utilizando filtro de lavagem contínua. TECNO-LóGICA v.15, n.1, p.6, 2011.

DOI: http://dx.doi.org/10.17058/tecnolog.v15i1.2162

MOREDJO, A.. Avaliação dos efeitos das atividades humanas sobre o estado trófico dos açudes paraibanos, com ênfase na utilização da comunidade zooplanctônica como bioindicador. Dissertação (Mestrado em Desenvolvimento e Meio Ambiente) - Universidade Federal da Paraíba, João Pessoa, 1998.

OLIVEIRA, Y. Q.; RONDON, O. C.. Diagnóstico da gestão de lodo de estação de tratamento de água em Mato Grosso do Sul. Interações (Campo Grande), v.17, p.687-698, 2016. DOI: http://dx.doi.org/10.20435/1984-042X-2016-v.17-n.4(11)

PÁDUA, V. L.; DI BERNARDO, L.. Método comparativo do tamanho dos flocos formados após coagulação com sulfato de alumínio e cloreto férrico. In: CONGRESSO INTERAMERICANO DE ENGENHARIA SANITÁRIA E AMBIENTAL, 27. Cancún, 2002.

PIANTÁ, C. A. V.. Emprego de coagulantes orgânicos naturais como alternative ao uso do sulfato de alumínio no tratamento de água. Monografia (Trabalho de conclusão de curso de Engenharia Civil) - Universidade Federal do Rio Grande do Sul, Porto Alegre, 2008.

POUMAYE, N.; MABINGUI, J.; LUTGEN, P.; BIGAN, M. Contribution to the clarification of surface water from the Moringa oleifera: Case M'Poko River to Bangui, Central African Republic. Chemical Engineering Research and Design. Elmsford, v.90, p.2346-2352, 2012. DOI: http://dx.doi.org/10.1016/j.cej.2015.04.045

RÔLA, A. K. K.; SANTANA, H. F.; CASTRO, G. M.; SOUZA, J. J. L. L.. Avaliação da eficiência de coagulantes comerciais para aplicação em sistemas de tratamento de água. Journal of Chemical Engineering and Chemistry, v.2, n.3, p.14-33, 2016. DOI: https://doi.org/10.18540/jcecvl2iss3pp014-033

ROSALINO, M. R. R.. Potenciais efeitos da presença de alumínio na água de consumo humano. Dissertação (Mestrado em Engenharia do Ambiente) - Faculdade de Ciências e Tecnologia, Universidade Nova de Lisboa, Lisboa, 2011. 
SANTOS, H. R.; PRADO, G. S.; VIDAL, C. M. S.; MORUZZI, R. B.; CAMPOS, J. R.. Aplicabilidade das técnicas de determinação de tamanho de partículas em sistemas de tratamento de água e esgoto sanitário. Engenharia Sanitaria e Ambiental, v.9, n.4, p.291-300, 2004. DOI:

http://dx.doi.org/10.1590/S1413-41522004000400005

SÁNCHEZ-MARTÍN, J.; BELTRÁN-HEREDIA, J.; PEREZ, J. A. Improvement of the Flocculation Process in Water Treatment by Using Moringa oleifera Seeds. Brazilian Journal of Chemical Engineering, v.29, n.3, p.495-501, 2012. DOI: http://dx.doi.org/10.1590/S0104-66322012000300006

TREVISAN, T. S.. Coagulante Tanfloc SG como Alternativa ao uso de Coagulantes Químicos no Tratamento de Água na ETA Cafezal. Monografia (Trabalho de Conclusão de Curso de Engenharia Ambiental). Universidade Tecnológica Federal do Paraná, Londrina, 2014.
TSUYUGUCHI, B. B.. Macrodrenagem e ocupação do solo no município de campina grande: caracterização, simulação e análises sistêmicas. Dissertação (Mestrado em Engenharia Civil e Ambiental) - Universidade Federal de Campina Grande, Campina Grande, 2015.

WANG, C.-T.; HU, J.-L.; CHOU, W.-L.; KUO, Y.-M.. Removal of color from real dyeing wastewater by Electro-Fenton technology using a three-dimensional graphite cathode. Journal of Hazardous Materials, v.152, n.2, p.601-606, 2008. DOI: http://doi.org/10.1016/j.jhazmat.2007.07.023

WHO. World Health Organization. Guidelines for Drinkingwater Quality. 3 ed. Geneva, 2004.

YIN, C. Y.. Emerging usage of plant-based coagulants for water and wastewater treatment. Process Biochemistry, v.45, n.9, p.1437-1444, 2010. DOI: http://doi.org/10.1016/j.procbio.2010.05.030

A CBPC - Companhia Brasileira de Produção Científica (CNPJ: 11.221.422/0001-03) detém os direitos materiais desta publicação. Os direitos referem-se à publicação do trabalho em qualquer parte do mundo, incluindo os direitos às renovações, expansões e disseminações da contribuição, bem como outros direitos subsidiários. Todos os trabalhos publicados eletronicamente poderão posteriormente ser publicados em coletâneas impressas sob coordenação da Sustenere Publishing, da Companhia Brasileira de Produção Científica e seus parceiros autorizados. Os (as) autores (as) preservam os direitos autorais, mas não têm permissão para a publicação da contribuição em outro meio, impresso ou digital, em português ou em tradução. 